\title{
Cold pulse and rotation reversals with turbulence spreading and residual stress
}

\author{
Hariri, F.; Naulin, Volker; Rasmussen, Jens Juul; Xu, G. S.; Yan, N.
}

Published in:

Physics of Plasmas

Link to article, DOI:

$10.1063 / 1.4951023$

Publication date:

2016

Document Version

Publisher's PDF, also known as Version of record

Link back to DTU Orbit

Citation (APA):

Hariri, F., Naulin, V., Rasmussen, J. J., Xu, G. S., \& Yan, N. (2016). Cold pulse and rotation reversals with turbulence spreading and residual stress. Physics of Plasmas, 23(5), [052512].

https://doi.org/10.1063/1.4951023

\section{General rights}

Copyright and moral rights for the publications made accessible in the public portal are retained by the authors and/or other copyright owners and it is a condition of accessing publications that users recognise and abide by the legal requirements associated with these rights.

- Users may download and print one copy of any publication from the public portal for the purpose of private study or research.

- You may not further distribute the material or use it for any profit-making activity or commercial gain

- You may freely distribute the URL identifying the publication in the public portal

If you believe that this document breaches copyright please contact us providing details, and we will remove access to the work immediately and investigate your claim. 


\section{AIP | Physics of

Cold pulse and rotation reversals with turbulence spreading and residual stress

F. Hariri, V. Naulin, J. Juul Rasmussen, G. S. Xu, and N. Yan

Citation: Physics of Plasmas 23, 052512 (2016); doi: 10.1063/1.4951023

View online: http://dx.doi.org/10.1063/1.4951023

View Table of Contents: http://scitation.aip.org/content/aip/journal/pop/23/5?ver=pdfcov

Published by the AIP Publishing

\section{Articles you may be interested in}

Turbulence induced radial transport of toroidal momentum in boundary plasma of EAST tokamak

Phys. Plasmas 23, 062309 (2016); 10.1063/1.4953601

Onsager's symmetry relation and the residual parallel Reynolds stress in a magnetized plasma with electrostatic turbulence

Phys. Plasmas 21, 092503 (2014); 10.1063/1.4894675

Effect of parallel currents on drift-interchange turbulence: Comparison of simulation and experiment

Phys. Plasmas 19, 102301 (2012); 10.1063/1.4757977

Turbulence and intermittent transport at the boundary of magnetized plasmas

Phys. Plasmas 12, 062309 (2005); 10.1063/1.1925617

Impact of large scale flows on turbulent transport

AIP Conf. Proc. 669, 696 (2003); 10.1063/1.1594025

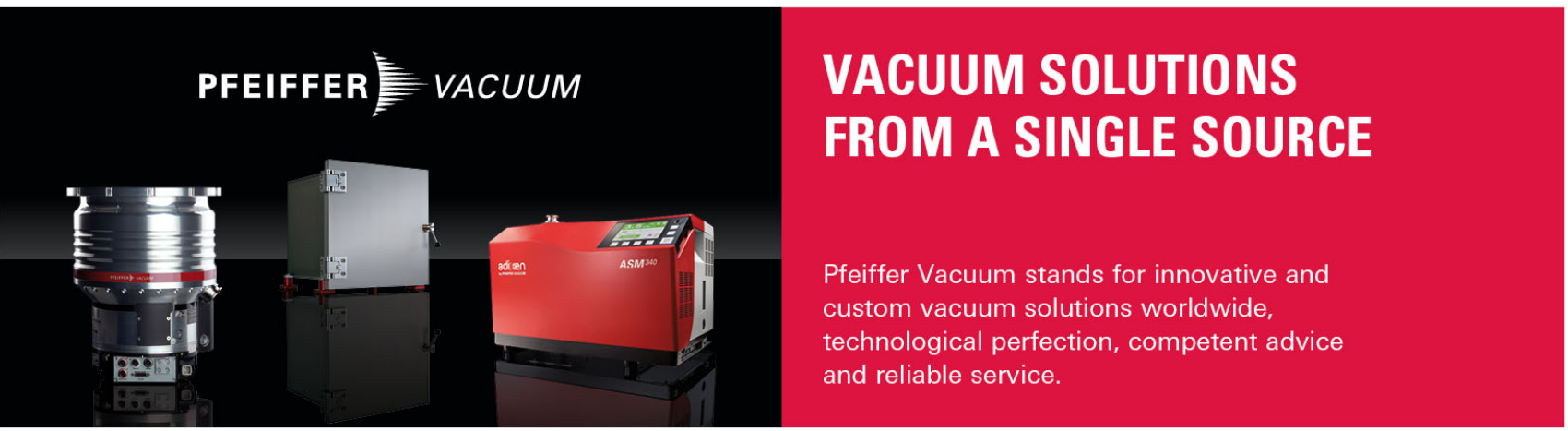




\title{
Cold pulse and rotation reversals with turbulence spreading and residual stress
}

\author{
F. Hariri, ${ }^{1}$ V. Naulin, ${ }^{2}$ J. Juul Rasmussen, ${ }^{2}$ G. S. Xu, ${ }^{3}$ and N. Yan ${ }^{3}$ \\ ${ }^{1}$ École Polytechnique Fédérale de Lausanne (EPFL), Swiss Plasma Center (SPC), CH-1015 Lausanne. \\ Switzerland \\ ${ }^{2}$ Technical University of Denmark (DTU), Department of Physics, DK-2800 Kgs. Lyngby, Denmark \\ ${ }^{3}$ Institute of Plasma Physics, Chinese Academy of Sciences, ASIPP, Hefei, China
}

(Received 29 February 2016; accepted 9 May 2016; published online 20 May 2016)

\begin{abstract}
Transport modeling based on inclusion of turbulence spreading and residual stresses shows internal rotation reversals and polarity reversal of cold pulses, with a clear indication of nonlocal transport effects due to fast spreading in the turbulence intensity field. The effects of turbulence spreading and residual stress are calculated from the gradient of the turbulence intensity. In the model presented in this paper, the flux is carried by the turbulence intensity field, which in itself is subject to radial transport effects. The pulse polarity inversion and the rotation profile reversal positions are close to the radial location of the stable/unstable transition. Both effects have no direct explanation within the framework of classical transport modeling, where the fluxes are related directly to the linear growth rates, the turbulence intensity profile is not considered and the corresponding residual stress is absent. Our simulations are in qualitative agreement with measurements from ohmically heated plasmas. Rotation reversal at a finite radius is found in situations not displaying saturated confinement, which we identify as situations where the plasma is nearly everywhere unstable. As an additional and new effect, the model predicts a perturbation of the velocity profile following a cold pulse from the edge. This allows direct experimental confirmation of both the existence of residual stress caused by turbulence intensity profiles and fundamental ideas of transport modeling presented here. Published by AIP Publishing.

[http://dx.doi.org/10.1063/1.4951023]
\end{abstract}

\section{INTRODUCTION}

Transport modeling is important for magnetically confined fusion plasma as it allows, on the one hand, predictions of the fusion performance and, on the other hand, serves machine safeguarding. Transport models used also to predict ITER performance are building on heavily reduced physics, often based on local quasi-linear approaches to turbulence, to be able to allow profiles evolution over many confinement times. To estimate the turbulence driven fluxes, the local instability rate of micro-instabilities is used together with their spatial scales. A large number of such models are in operation, at times also based on the parametrized detailed outcome of complex first-principles local gyrokinetic simulations of plasma turbulence. ${ }^{1}$ In most situations, transport modeling shows reasonably good results when compared to experiment particularly for the core region, which constitutes a clear success of the framework. However, a number of dynamic transport effects in magnetically confined plasmas are not understood or even reproducible in this approach to transport modeling. One of these is connected with short dynamic transport events. In experiments, short time perturbations to the plasma are triggered by cooling the edge of the plasma using a burst of influx of heavy impurities triggered by laser ablation. ${ }^{2}$ The resulting cold pulse is observed to traverse the plasma with speeds much higher than predicted from transport modeling. Often a reversal in the cold pulse polarity is observed, i.e., a temperature increase at certain plasma radius follows the cold pulse trigger instead of a cooling. Another difficult to capture feature is the rotation behavior in the absence of large momentum sources in the plasma, such as caused by neutral beam injection, and thus dominating ohmically or RF-heated plasma scenarios. In these cases, the plasma rotation is dominated by the boundary values at the scrape-off layer and internal redistribution of momentum. This internal redistribution due to turbulent momentum flux can only be partly cast into the standard formulation used in transport codes, namely, into diffusive terms and pinch terms, the remaining flux being called the residual stress. ${ }^{3}$ This separation of transport into diffusion and pinch is one of the unifying features of transport models. Here, we investigate the general, qualitative behavior of a model which not only for the momentum transport but also for heat transport breaks with this approach. Instead of the transport being related to the turbulence growth rate, which is locally evaluated from plasma properties and its gradients, we consider the plasma instabilities to feed into a turbulence intensity field. The energy is thus first transferred from the driving gradients to the turbulence velocities, and the turbulence intensity field is then responsible to drive the transport. However, the turbulence intensity field itself can spread out, an effect known as turbulence spreading or turbulence penetration. ${ }^{4-9}$ In the absence of turbulence spreading, this approach is mostly equivalent to the classical one, as the growth rates of the instabilities are large compared to transport timescales, and local saturation of the turbulence intensity field happens nearly instantaneous. By including the 
turbulence intensity field, this new approach has two implications. One is that we have access to the turbulence intensity field and can use its properties to determine, for example, residual stresses. ${ }^{3,10}$ More importantly, with turbulence spreading, as the turbulence intensity field becomes a transported quantity, we break with the locality inherent in classical transport models and add with the turbulent spreading non-locality in a transparent way. In the following, we discuss the layout and qualitative results for a prototypical turbulence spreading transport model in Sec. II. In Sec. III, we discuss the steady state properties of the model using the predicted temperature profiles and discuss the effect of residual stress, driven by the turbulence intensity gradient, on the rotation profiles. We then turn to dynamical effects in Sec. IV, where we consider cold pulse propagation. We show that polarity reversal of cold pulses is a possibility. The pulse in turbulence intensity travels ahead of the cold pulse front-as observed in the temperature-what is demonstrated to be the cause of the inherent nonlocality of the plasma response. This can lead to much faster reactions of the plasma to the cold pulse than anticipated. We also discuss the effect the cold pulse has on the rotation profile. This effect is absent from classical transport modeling and allows for a direct experimental test of the ideas presented in this paper and also for the direct experimental proof of the turbulence intensity profile driven by residual stress, which was proposed in Ref. 11. Finally, in Sec. V, we discuss the main results and conclusions.

\section{TURBULENCE SPREADING TRANSPORT MODEL}

The turbulent flux in standard transport models is proportional to the growth rate, determined from the local plasma properties such as local gradients and values of the plasma variables. In this paper, we consider an alternative approach in which the instability drives the turbulence intensity field, $I$, itself subject to transport. The turbulent fluxes are related to $I$ and are thus only indirectly coupled to the growth rate. This allows to take into account a number of effects usually absent in standard transport models. The transport of the turbulence intensity field, named turbulence spreading, can lead to nonlocality as turbulence is transported from unstable into stable regions. ${ }^{12}$ Transport models with turbulence spreading have been proposed in Refs. 7, 8, and $12-14$, and conditions under which the perturbation in the turbulence profile could travel at the required high speed from the edge to the core have been established in Ref. 15 .

In the model proposed in Ref. 12, the turbulence spreading is modeled by a reaction-diffusion partial differential equation of the Fisher-Kolmogorov-Petrovskii-Piskunov (FK) type,${ }^{16}$ an approach also used in Ref. 17. In the present investigation, we will apply a similar approach. The turbulent transport is determined from the turbulence intensity field, but accounts also for an approximated phase relationship between velocity fluctuations and the quantity transported.

In the model we propose, we consider the evolution of the temperature field, the turbulence intensity field and its spreading, and add to it the evolution of toroidal momentum. One residual stress component has been derived to be due to the gradient of the turbulence intensity field $I .{ }^{10,11}$ Including this residual stress is only possible in a transport model where the turbulence intensity is accessible, and thus, this constitutes a unique aspect of this model.

\section{A. Motivation of the model}

We consider a 1D transport model with the profiles equivalent to flux surface averaged quantities. Differential operators are in the radial direction only. We assume the density profile to be frozen and constant, and propose the following model equations for the profiles of turbulent intensity $I \propto|\phi|^{2}$, with $\phi$ being the electric potential fluctuations, temperature $T$, and toroidal momentum $M$ :

$$
\begin{gathered}
\frac{\partial I}{\partial t}=\nabla \cdot\left[D_{0} I \nabla I\right]+\gamma I-\left(\beta I^{2}\right) I, \\
\frac{\partial T}{\partial t}=-\nabla \cdot\left(\vec{q}+\chi_{0} \nabla T\right)+S(r), \\
\frac{\partial M}{\partial t}=\nabla \cdot\left[D_{M} \nabla M+V_{\text {Pinch }} M+\mathcal{R}\right],
\end{gathered}
$$

with

$$
D_{0} I=D_{\text {turb }},
$$

and with the turbulence spreading being the essential nonlinearity of the FK type of equation. The FK type nonlinearity, allowing for front propagation, can also be interpreted as a turbulent diffusion coefficient $D_{t u r b}$, which is dependent on the turbulence level itself. The temperature $T$ evolves due to a localized source $S(r)$ and the divergence of the turbulent heat flux $\vec{q}$. The constant diffusivities in the temperature and momentum equations signify classical/neoclassical transport. The turbulent intensity is created from an instability with growth rate $\gamma$ and is limited in amplitude by a nonlinear saturation proportional to $\beta$. It is worthwhile to note that the turbulence intensity is positive definite as the cubic nonlinear term in Eq. (1) cannot cause a change of sign. We use a simple expression to determine the growth rate from the deviation $d$ of the temperature gradient

$$
\kappa_{T}=-\frac{\nabla T}{T},
$$

from a critical value $\kappa_{c}$ :

$$
\begin{gathered}
d=\kappa_{T}-\kappa_{c}, \\
\gamma=\lambda(\operatorname{sign} d)\left[\kappa_{T}-\kappa_{c}\right],
\end{gathered}
$$

$\lambda(-)$ being $5 \%$ of $\lambda(+)$, resulting in an approximate shape for the growth rate including the stable, damped branch of the whole dispersion relation. Opposite to classical transport modelling, it is important in the context of turbulence spreading to consider as well the damped branch of the dispersion relation, as we have to deal with turbulence intensity that has been convected into stable regions of the considered plasma. In these stable regions, the turbulence energy is dissipated and can drive the profile towards the marginally stable one from below. Conversely in unstable regions the 
energy put into the turbulence is taken from the free energy available in the supercritical gradient and thus leads to the release of heat flux in this model. Again this is absent in models without the effects of turbulent spreading, but recovers phenomena observed in direct numerical simulations ${ }^{12}$ and ideas of turbulent transport leading to turbulent equipartition (TEP). ${ }^{4,18-20}$ Following the arguments in Ref. 12, we use the growth rate $\gamma$ to introduce an approximate phase relationship between the turbulent velocity and the temperature fluctuations $\tilde{T}$ as $\tanh (\gamma)$. This limits the phase to values between -1 and 1 . The anomalous transport is then given by

$$
\vec{q}=\langle\tilde{T} \tilde{v}\rangle \hat{x}=C \tanh (\gamma)\left\langle\sqrt{\tilde{v}^{2}} \sqrt{\tilde{T}^{2}}\right\rangle \hat{x} .
$$

In this expression, we approximate the velocity fluctuations $\tilde{v}$ by the electrostatic potential $|\tilde{\phi}|$ for the low frequency turbulence, considered that fluctuating velocity is basically given by the $E \times B$ velocity and thus strongly related to $\phi$. We further assume that the level of temperature fluctuations is proportional to the level of turbulence, that is, $\tilde{T} / T \sim|\tilde{\phi}| \sim \sqrt{I}$. Absorbing all proportionalities into the constant $C$, we arrive at the magnitude of the flux

$$
q=C \tanh (\gamma) I T .
$$

The anomalous transport is in this model proportional to the energy $I$ in the turbulence. It additionally scales with the average cross-phase between temperature fluctuations $\tilde{T}$ and turbulent velocity fluctuations $\tilde{v}$, which we have taken to be proportional to the growth rate $\gamma$. The latter is proportional to the deviation from the critical profile as seen from Eq. (7) and introduces the proportionality of flux to the gradient that is usually found in classical transport models. If the system is nowhere above the critical gradient and in the absence of a source, initial turbulent noise dies away, and transport is due to (neo)-classical, i.e., collisional effects and confinement geometry. Turbulence is exited as the threshold gradient $\kappa_{c}$ is exceeded. The system will relax to a temperature profile close to the marginally stable one. The ability of the system to create turbulent flux once the critical gradient is exceeded is related to the stiffness of that profile. In this model, besides the turbulence intensity $I$, also the phase $\tanh \gamma$ determines the capability of the system to generate anomalous transport and thus the profile stiffness.

The main difference with classical transport modeling is that we here also consider the turbulent transport in regions where the turbulence is damped, as these regions are receiving a non-zero level of turbulent intensity by turbulence spreading, i.e., the transport of the turbulence intensity field, $I$, into these otherwise quiescent regions. Here, the turbulence intensity field, $I$, causes transport in the up-gradient direction. If the growth rate is negative, the phase relationship between temperature and velocity fluctuations changes. The turbulent intensity (or energy, respectively) is depleted on steepening up the gradient towards the marginal one, revealing energy conservation. It is necessary to note that no global up-gradient transport is possible. Globally, the transport is always down-gradient, only part of the transport is directed the other way and can contribute to a pinch effect, as the turbulence energy is taken from decreasing the gradient. Finally, the parameter $C$ also includes the proportionality between velocity and temperature fluctuations, and absorbs the square of the spatial scale of the turbulence in analogy to standard mixing length arguments. ${ }^{21}$

The final ingredient into the transport model is a simple inclusion of the transport of toroidal momentum $M$ referenced by Eq. (3). It parametrises the turbulent momentum transport by a diffusion $D_{M}$ and a pinch velocity $V_{\text {Pinch }}$, including residual stress $\mathcal{R}$. It is worthwhile to note that the residual stress $\mathcal{R}$ appears as a flux and not as a source, thus arranging momentum redistribution, but not adding net momentum. It has been found that residual stress is driven by a number of effects, such as up-down asymmetry, poloidal flow shear, plasma shaping, and others. ${ }^{10}$ Here, we consider the mechanism most important in the context investigated, residual stress drive by the turbulence intensity gradient ${ }^{11}$ formulated, with $\beta$ being a proportionality parameter, as:

$$
\mathcal{R}=\beta \frac{\nabla I}{I} .
$$

We can now study the qualitative effect of turbulence spreading and flow generation by residual stress in a paradigmatic setup. The feedback of the generated flow shears on the turbulent transport is ignored here, but could be a simple extension of the model.

\section{B. Numerical implementation}

The model equations are implemented in cylindrical geometry in $1 D$, using second order centered finite differences for the differential operators. The equations for positive definite quantities as $I$ and $T$ are implemented in terms of the logarithm of the quantities. Time stepping is provided by a fourth order embedded Gear algorithm following the work by Karniadakis, where the diffusive terms are treated implicitly. ${ }^{22}$ The occurring Helmholtz problems are solved using a direct Gaussian solver. The time step is adjusted to the level of turbulence generated, which varies on short timescales as the growth rates can be large compared to the transport timescales, and is of order $1 e-5$ for the situations considered here. The boundary conditions at $r=0$ are zero gradient and zero fluxes, and at $r=1$, we keep the temperature and momentum fixed to reference values 0.785 and 2 , respectively. The critical gradient is fixed to 1 and $\lambda(+)$ is kept constant at 0.85. An inward momentum pinch $V_{\text {Pinch }}$ is set to $10^{-2}$ throughout the simulations. The turbulence intensity has zero turbulence flux at $r=1$. Initial conditions are low level white noise in all quantities, and in the temperature equation, the heat source $S(r)=h r^{2}$ is used where $h$ is a free factor and $h / 3$ denotes the total heating power varied for our purposes between 0 and 10. The diffusivities are kept constant throughout the simulations at $D_{M}=10^{-2}$ and for the classical heat transport at $\chi_{0}=10^{-3}$. For numerical reasons, we put an additional artificial diffusion term $\chi_{t}=10^{-5}$ on the turbulence intensity equation. The parameters are chosen in a way that the temperatures considered could be easily translated to more practical values of $\mathrm{keV}$, and to facilitate 
reading the paper, we sometimes use this reference temperature. The radial simulation domain is normalized to one.

\section{STEADY-STATE PROPERTIES OF THE MODEL}

An important feature of the model is the effect of turbulence spreading onto the transport in the stable region of the plasma. The general chain of events is as follows: Temperature increases with heating. Once the critical gradient is exceeded, turbulence intensity is generated and the temperature profile stays more or less, depending on stiffness, fixed to the critical gradient. The difference between the model with turbulence spreading and the one without is minimal in the unstable region for $r>0.5$. In the stable region for a model without turbulence spreading, the transport is carried by the diffusive flux and the temperature gradient at $r$ adjusts accordingly to the integrated flux occurring at position $r$. This is illustrated in Fig. 1, where the transition from stable to unstable occurs at around $r=0.5$. The simulation with turbulence spreading $\left(D_{t u r b}=1\right)$ shows the penetration of turbulence from the unstable region into the stable one $(r<0.5)$. Here, the turbulence causes an up-gradient flux which is countered by a diffusive down-gradient flux in steady state, such that the heating power integrated to position $r$ is transported out in steady state. The resulting profile is, however, much steeper and approaches the marginally critical profile from below. As the heat flux that is carried at a radial position is monotonously increasing as a function of radius, the level of turbulence needed to carry that flux increases with radius as well. The turbulence spreading arranges for the turbulence intensity field to penetrate into the stable region, which results in a background level of turbulence, as shown in Fig. 2. Note that in the stable region $r<0.5$, the direction of the turbulent heat flux is reversed and mediated by the factor $\lambda(-)$. Increasing the heating (without changing the heating profile) lets the plasma become unstable at increasingly smaller radial positions. Clearly, the steady state gradient has to balance the integrated heating power, and there is a critical heat flux at which the classical heat transport prescribes a gradient exceeding the critical one

$$
\Gamma_{c}=\chi_{0} \kappa_{c} .
$$

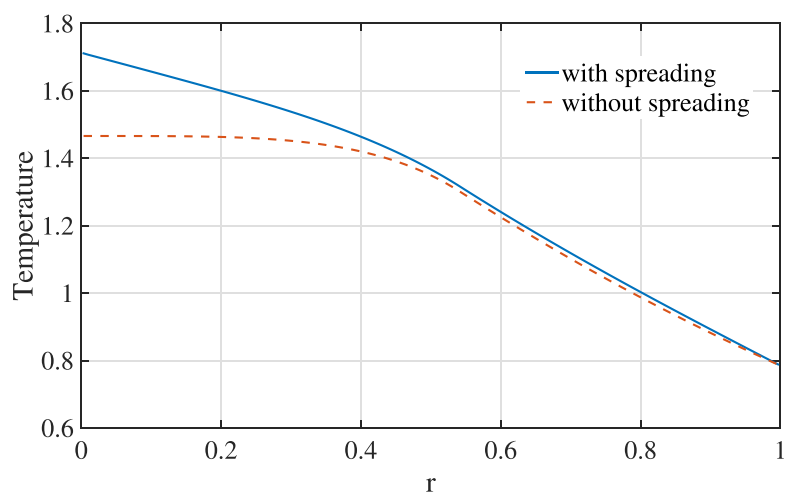

FIG. 1. Temperature as a function of radius determined with spreading $D_{\text {turb }}=1$ (blue sold line), and without turbulence spreading $D_{\text {turb }}=0$ (red dashed line) and at $h=0.05$.

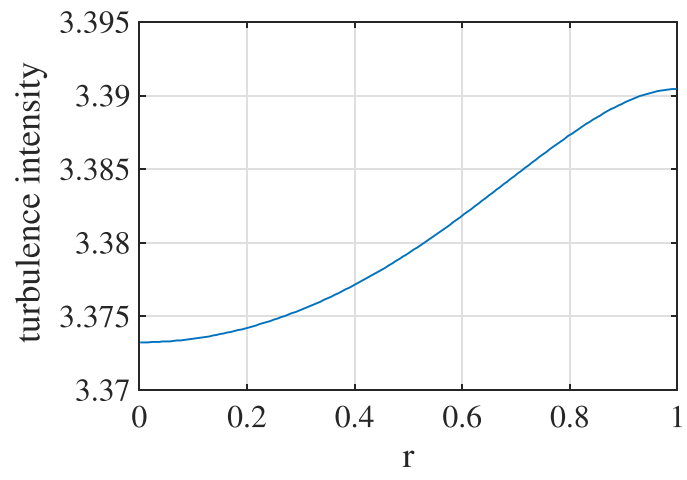

FIG. 2. Turbulence intensity profile in steady state, for the same parameters as in Figure 1.

This radius, $r_{c}$, can be determined from the condition

$$
\Gamma_{c}=\int_{0}^{r_{c}} S(r) d r
$$

and the dependence of $r_{c}$ from the heating is shown in Fig. 3. At sufficiently high heating, the whole profile will be unstable and the stable/unstable transition will ultimately disappear. In this context, it is worthwhile to consider the overall confinement properties exhibited by this model. At very low heating powers, the turbulence is weak and the distance from the marginally stable profile stays comparatively large. Increasing heating from this level brings the profile quickly closer to the marginal one, and the central core temperature increases rapidly. After a certain level of turbulence has been triggered (see Fig. 4, at heating $h$ exceeding 0.1), the profile is already close to the marginal one, and any further improvement of core temperature, which is for monotonous profiles related to the stored energy, is only related to the level of stiffness found in the model. We believe that this behavior is similar to the characterization of ohmic confinement into the regimes of saturated ohmic confinement (SOC) and linear ohmic confinement (LOC). ${ }^{23}$ LOC corresponds to the situation where the profile is still sufficiently away from the marginal critical one, so that additional heating power still results in an increase in confined energy or core

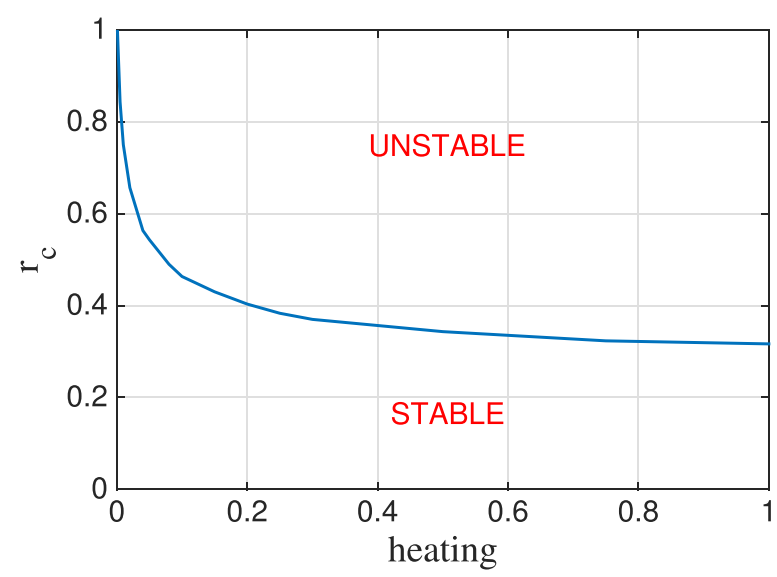

FIG. 3. Radial position of the transition from stable to unstable plasma as the heating $h$ is increased. The transition occurs where the flux, critical flux $\Gamma_{c}$, is exceeded. 


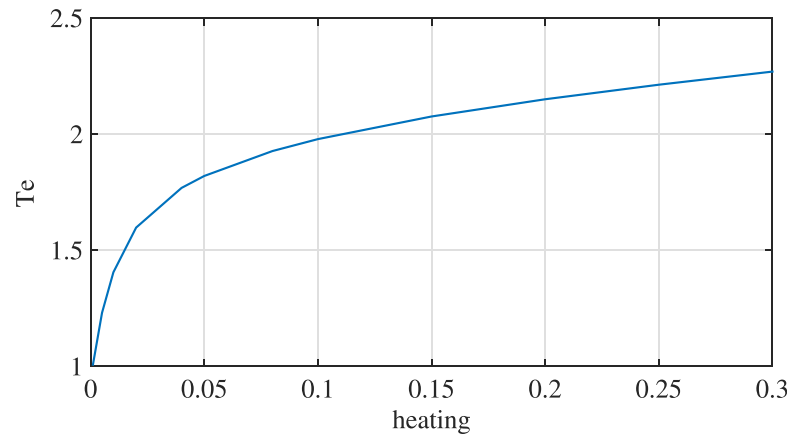

FIG. 4. Evolution of core temperature with heating $h$ showing a saturation at high heating powers.

temperature, respectively. When the system develops stronger levels of turbulence and the profile gets close to the marginal one, we arrive ultimately in the SOC state. SOC corresponds to a situation where most of the plasma is unstable. In experiments, relevant instability would be of the Ion Temperature Gradient (ITG) instability type. The nature of the transition will also depend on the heating profile, as a central heating profile will ensure that the critical gradient is exceeded at smaller radius than a distributed heating profile. Figure 5 shows the stationary rotation profile determined by three factors: the first one is the boundary value at the scrape-off-layer $(r=1)$, the second is the pinch velocity, and the third is the finite flux caused by the residual stress. With a transition from stable to unstable in the plasma domain, there is a steep gradient in the turbulence intensity profile and thus also a large localized residual stress. The residual stress in steady state is shown in Fig. 6. It is seen that the turbulence intensity profile $I$ creates a residual stress $\mathcal{R}$, which leads to a flux in momentum of finite size. This flux is important only for certain conditions. If the momentum fluxes caused by momentum sources in the plasma, or the momentum flux carried by an inward pinch from the non-zero momentum boundary condition at $r=1$ exceeds this residual stress $\mathcal{R}$, the effect of it becomes negligible. Thus, for large values of the turbulence spreading parameter resulting in a

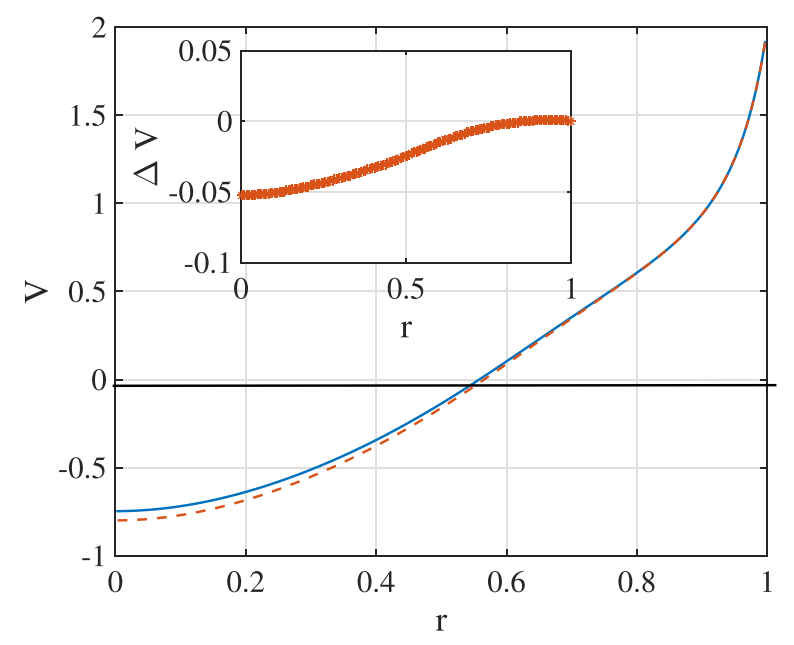

FIG. 5. Velocity profile in steady-state just before the cold pulse (solid blue line) and 300 time units after the cold pulse (dashed red line) for the parameters discussed in the text. The inset shows the velocity difference.

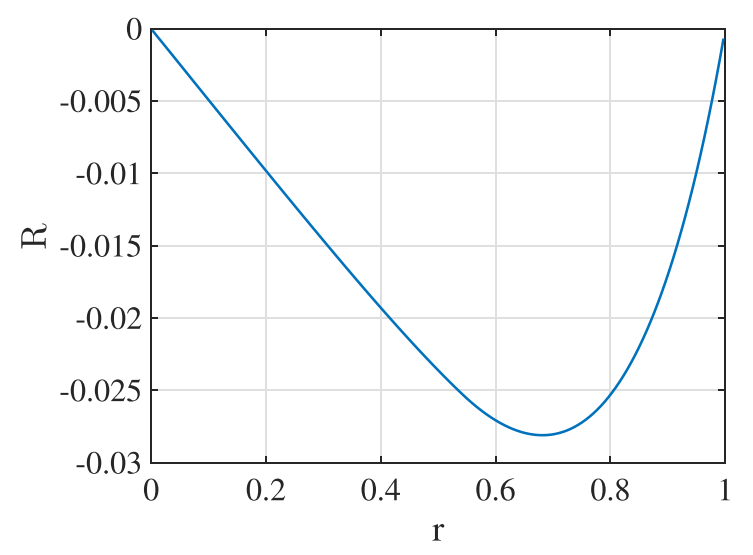

FIG. 6. Residual stress in steady-state.

flat turbulence intensity profile, or for situations where the plasma is nearly everywhere unstable, also resulting in a more flat turbulence intensity profile, we expect vanishing influence of the residual stress on the rotation profile. This latter situation we expect again in the SOC regime where the plasma is everywhere unstable and the turbulence intensity $I$ would consequently be absent of large gradients driving residual stress, coinciding with the observation that internal rotation reversal is often to be found absent in SOC discharges. ${ }^{23}$ In a situation, where the pinch flux from the boundary is similar to the residual stress flux, we, however, can expect effects. One is that the rotation can now change sign at some radial position in the plasma, an effect absent from usual transport modeling. Together with an assumed pinch velocity, this allows for significant core rotation velocities including zero crossing of the velocity profile. The effect of the residual stress will be somewhat, but not strictly, related to the transition from stable to unstable plasma conditions, and thus, we expect the rotation reversal also to take place in the vicinity of this transition, as can be seen from Fig. 5.

\section{DYNAMICAL PROPERTIES OF THE MODEL}

In experiments, cold pulses are used for perturbative transport experiments where the plasma is cooled down at the edge rapidly following laser ablation of metal impurities. It is known that the cold pulse propagates very fast into the plasma, faster than expected by usual transport analysis. ${ }^{9,24}$ Frequently, cold pulses trigger transient temperature increases in the plasma core. This phenomenon is known as cold pulse reversal ${ }^{23,25,26}$ and visualised in Figure 8. Cold pulse reversal has no well established explanation in classical transport modeling. We perform simulations with heating amplitude $h=0.05$ and anomalous coupling $C=0.01$. In the absence of turbulence spreading $\left(D_{\text {turb }}=0\right)$, indeed, no reversal of the cold pulse is observed in the simulation. Figure 7 shows this situation. The cold pulse is initiated at time $t=0$ by reducing the boundary value for the temperature by $20 \%$. The fast inward propagation of the cold pulse is clearly visible in the unstable plasma region. Cold pulse propagation slows somewhat in the stable region, and after a few time units, the central temperature begins to drop. In 


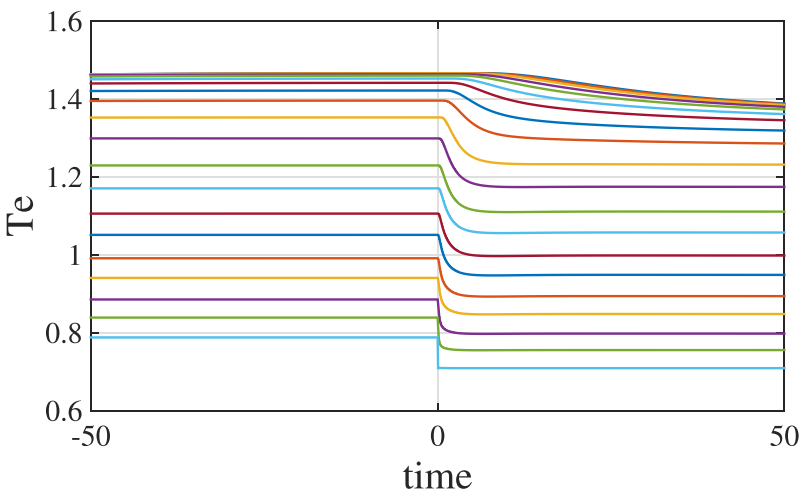

FIG. 7. Temperature traces (at 20 equidistant radial positions, from $r=0$ to $r=1$ and with values corresponding to temperature units in $\mathrm{keV}$ ) for the system without turbulence spreading $\left(D_{\text {turb }}=0\right)$. The fast inward propagation of the cold pulse through the unstable plasma region is seen. The cold pulse is triggered at $t=0$.

Figure 8 , the situation in case $D_{\text {turb }}=1$ is shown. With spreading, the profile approaches the critical one, and thus, the core temperature exceeds the one observed in the case without spreading, as was already established in Sec. III. In this situation, we also observe the fast propagation of the cold pulse through the unstable part of the radial domain. However, the cold pulse now triggers an intermediate increase in the core temperature. It is interesting to notice that the reversal happens, at its largest radial extent, in the vicinity of the transition from unstable to stable plasma conditions, but also that the increase in temperature starts on a timescale that is faster than the cold pulse related drop, seen in Figure 7.

To investigate this situation in more detail, we look at the radial position of the transition from stable to unstable plasma. We also determine the radial position of the cold pulse over time as the first radial position where the drop in temperature is larger than $0.2 \mathrm{eV}$, and correspondingly, the position of the cold pulse reversal as the outermost point where the increase in temperature is larger than $0.2 \mathrm{eV}$. The result is shown in Figure 9, showing an extremely fast propagation of the cold pulse front though the unstable region of the plasma from $r=1$ inwards to $r=0.55$. Here, the cold

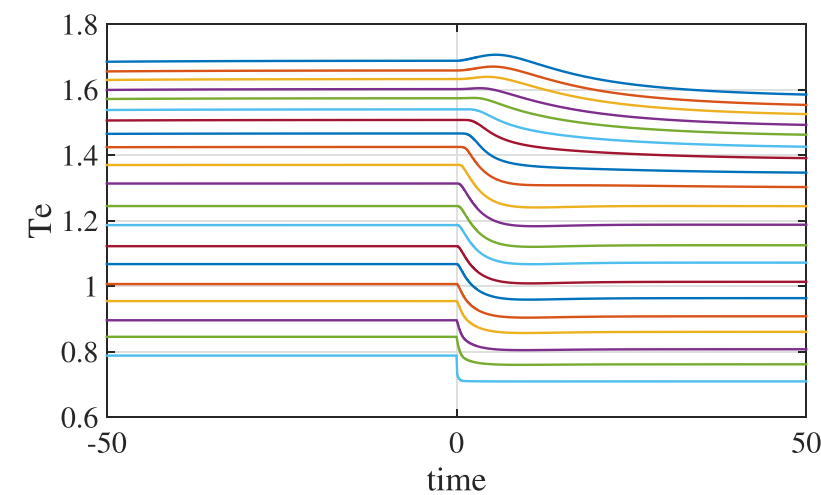

FIG. 8. Temperature traces in $\mathrm{keV}$ (at 20 equidistant radial positions as above) for the system with turbulence spreading $\left(D_{\text {turb }}=1\right)$. The fast inward propagation of the cold pulse through the unstable plasma region is seen. In addition, the cold pulse reversal leads to an intermediate core temperature increase. The cold pulse is triggered at $t=0$. pulse slows down and travels inwards with a much more reduced speed, while the stable unstable transition point is moved slightly inward. The reversal of the cold pulse is now seen to be an effect spreading inward from $r=0$ quickly and meeting the cold pulse front after one time unit. It is important to note that the reversal starts at a time very shortly after the cold pulse trigger. A first response at $r=0$ is already seen at $t=0.02$, roughly at a time where the extrapolated cold pulse front would have reached the center, if it had not slowed down after passing the stable/unstable transition point. This very fast response of the plasma to the cold pulse trigger is due to the turbulence front propagating inward.

In Figure 10, the turbulence in the core and at the edge is shown to react on a fast timescale to the cold pulse triggered at $t=0$. The turbulence intensity $I$ thus spreads the information of the cold pulse triggered throughout the whole volume of the plasma in a short time. The extra energy in the turbulence intensity field arranges for extra up gradient heatflux in the stable plasma region, leading to the observed cold pulse reversal. This exhibits, at the same time, a non-local response to the cold pulse. The temperature in the core changes without any information being passed along the temperature channel. Clearly, this effect cannot be captured by any model building on information being transported in the temperature channel alone. Thus, effects like this are conveniently modeled by employing non-local integral operators in the heat diffusion equation, see for example, Ref. 27.

It is instructive and interesting to look at the influence of the cold pulse on the rotation profile. The cold pulse triggers large perturbations in the turbulence intensity profile as shown in Figure 10. These changes in the turbulence intensity profile also change the residual stress derived from the intensity profile gradient and can therefore have an influence onto the rotation profile.

We show the velocity traces after the cold pulse trigger in Figure 11. The velocity profile shows a fast response to the cold pulse triggered. The timescale of the propagation of this response is the same as the propagation speed observed in the turbulence intensity profile perturbation. We observe a reversal of the polarity in the changes of the velocity profile due to the residual stress being a redistribution of momentum, but not a net source. As we are here in a situation where the residual stress is important compared to any other

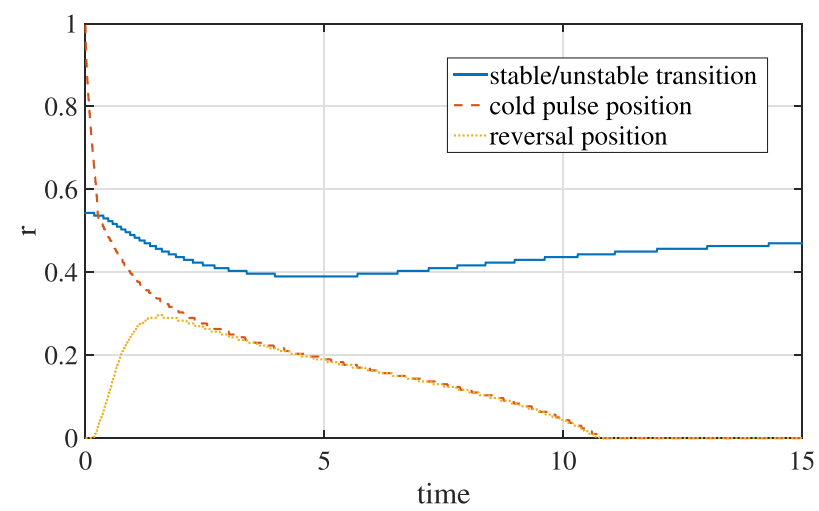

FIG. 9. Radial position of the stable/unstable transition, the cold pulse front, and the cold pulse reversal after the cold pulse trigger at $t=0$. 


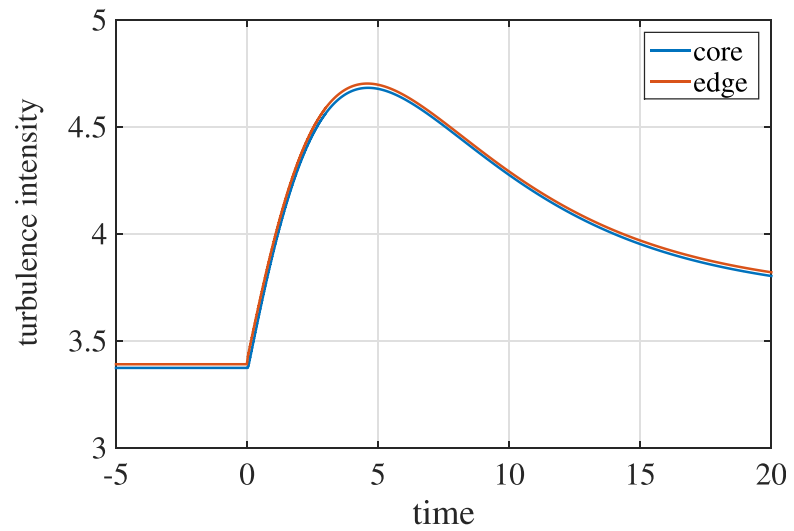

FIG. 10. Time trace of the core $r=0$ and edge value $r=1$ of the turbulence intensity profile. The turbulence intensity reacts very quickly to the cold pulse trigger at $t=0$.

momentum flux through the system, the velocity perturbations caused by the cold pulse are significant and potentially measurable in experiments, even though we cannot make quantitative predictions on the size of the effect. This would allow direct demonstration of the effect of turbulence intensity gradients on residual stresses.

\section{CONCLUSION}

In this contribution, we studied the properties of a transport model breaking with the local paradigm usually employed in transport investigations. Considering a relatively simple model including the effects of turbulence spreading and the impact of the turbulence intensity profile onto the residual stresses, we demonstrated fast cold pulse propagation and cold pulse polarity reversal. For the stationary profiles, the inclusion of turbulence spreading has nearly no effect, but in the stable plasma region, the profiles are driven towards the marginal one, thus arranging for near critical profiles over the whole extent of the plasma. The effects of turbulence spreading have also been recently investigated in gyrokinetic first principles simulations. At present, these simulations can mostly address small profile variations, but

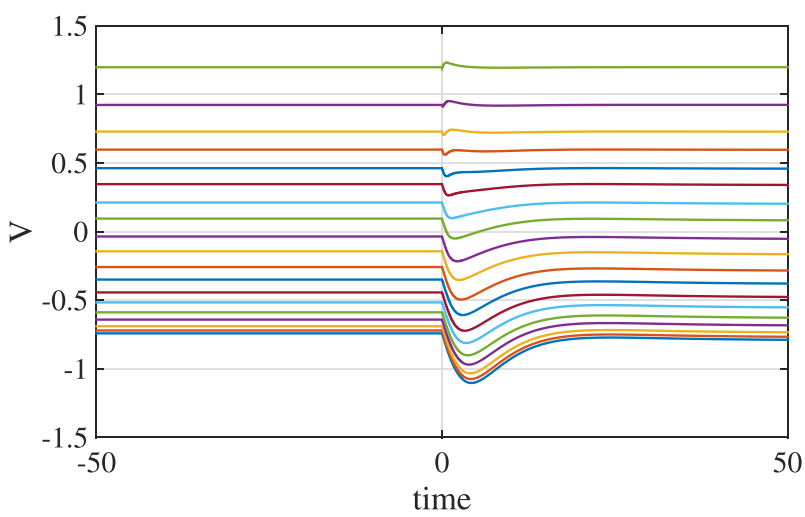

FIG. 11. Velocity traces at 20 equidistant radial positions after cold pulse trigger at $t=0$, for the standard parameters discussed above. The core is at large negative rotation velocities and the edge at large positive rotation velocities. Besides the zero crossing in the middle of the domain, a clear effect of the cold pulse on the temporal evolution of the velocities is seen. The propagation time of the effect is again related to the turbulence intensity profile changes. clearly show turbulence intensity front propagation. ${ }^{28-30} \mathrm{~A}$ fast speed of information propagation though the plasma is due to the turbulence intensity profile which, if observed by its consequences onto the temperature profile, would appear as a non-local effect. The reversal of the cold pulse is found to be connected to the stable/unstable transition, and a zero crossing of the rotation profile happens in the approximate region of this transition as well. We propose that the location of this transition point is responsible for the saturation of the confinement. In the saturated confinement, the whole plasma domain is filled with high levels of turbulence, and the profile, both in sub- and supercritical regions, is deviating only weakly from the critical profile. A new finding is that rapid turbulence intensity profile changes act on the residual stress. This results into changes in the velocity profile. This new effect potentially allows to experimentally verifying the existence of both turbulence spreading and turbulence intensity profile induced residual stresses, which are both absent from classical turbulence transport modeling. Such experiments would shed light onto the fundamental properties of turbulent transport and momentum redistribution in tokamaks, and be of primary interest.

${ }^{1}$ J. E. Kinsey, G. M. Staebler, J. Candy, R. E. Waltz, and R. V. Budny, "ITER predictions using the GYRO verified and experimentally validated trapped gyro-landau fluid transport model," Nucl. Fusion 51(8), 083001 (2011).

${ }^{2}$ P. Mantica and F. Ryter, "Perturbative studies of turbulent transport in fusion plasmas," Comptes Rendus Physique 7(6), 634-649 (2006).

${ }^{3}$ P. H. Diamond, C. J. McDevitt, Ö. D. Gürcan, T. S. Hahm, W. X. Wang, E. S. Yoon, I. Holod, Z. Lin, V. Naulin, and R. Singh, "Physics of nondiffusive turbulent transport of momentum and the origins of spontaneous rotation in tokamaks," Nucl. Fusion 49(4), 045002 (2009).

${ }^{4}$ V. Naulin, J. Nycander, and J. Juul Rasmussen, "Equipartition and transport in two-dimensional electrostatic turbulence," Phys. Rev. Lett. 81(19), 4148 (1998)

${ }^{5} \mathrm{~N}$. H. Brummell, T. L. Clune, and J. Toomre, "Penetration and overshooting in turbulent compressible convection," Astrophys. J. 570(2), 825 (2002).

${ }^{6} \mathrm{Z}$. Lin, S. Ethier, T. S. Hahm, and W. M. Tang, "Size scaling of turbulent transport in magnetically confined plasmas," Phys. Rev. Lett. 88(19), 195004 (2002).

${ }^{7}$ T. S. Hahm, P. H. Diamond, Z. Lin, K. Itoh, and S. I. Itoh, "Turbulence spreading into the linearly stable zone and transport scaling," Plasma Phys. Controlled Fusion 46(5A), A323 (2004).

${ }^{8}$ Ö. D. Gürcan, P. H. Diamond, T. S. Hahm, and Z. Lin, "Dynamics of turbulence spreading in magnetically confined plasmas," Phys. Plasmas 12(3), 032303 (2005).

${ }^{9}$ V. Naulin, J. J. Rasmussen, P. Mantica, and D. del Castillo-Negrete, "Fast heat pulse propagation by turbulence spreading," in The Japan Society of Plasma Science and Nuclear Fusion Research Proceedings (2009).

${ }^{10}$ P. H. Diamond, Y. Kosuga, Ö. D. Gürcan, C. J. McDevitt, T. S. Hahm, N. Fedorczak, J. E. Rice, W. X. Wang, S. Ku, J. M. Kwon et al., "An overview of intrinsic torque and momentum transport bifurcations in toroidal plasmas," Nucl. Fusion 53(10), 104019 (2013).

${ }^{11}$ Ö. D. Gürcan, P. H. Diamond, P. Hennequin, C. J. McDevitt, X. Garbet, and C. Bourdelle, "Residual parallel reynolds stress due to turbulence intensity gradient in tokamak plasmas," Phys. Plasmas 17(11), 112309 (2010).

${ }^{12}$ V. Naulin, A. H. Nielsen, and J. Juul Rasmussen, "Turbulence spreading, anomalous transport, and pinch effect," Phys. Plasmas 12(12), 122306 (2005).

${ }^{13}$ R. E. Waltz and J. Candy, "Heuristic theory of nonlocally broken gyroBohm scaling," Phys. Plasmas 12(7), 072303 (2005).

${ }^{14}$ Ö. D. Gürcan, P. H. Diamond, and T. S. Hahm, "Nonlinear triad interactions and the mechanism of spreading in drift-wave turbulence," Phys. Rev. Lett. 97(2), 024502 (2006). 
${ }^{15}$ X. Garbet, Y. Sarazin, F. Imbeaux, P. Ghendrih, C. Bourdelle, Ö. D. Gürcan, and P. H. Diamond, "Front propagation and critical gradient transport models," Phys. Plasmas 14(12), 122305 (2007).

${ }^{16}$ A. N. Kolmogorov, I. G. Petrovsky, and N. S. Piskunov, "A study of the equation of diffusion connected with an increase in the quantity of matter and its application to a biological problem," Bull. Univ. Moscow 1(6), 1-25 (1937).

${ }^{17}$ P. Migliano, R. Buchholz, S. R. Grosshauser, W. A. Hornsby, A. G. Peeters, and O. Stauffert, "Turbulence spreading in gyro-kinetic theory," Nucl. Fusion 56(1), 014002 (2016).

${ }^{18}$ M. B. Isichenko and V. V. Yankov, "Turbulent equipartitions in twodimensional drift convection," Phys. Rep. 283(1), 161-175 (1997).

${ }^{19}$ T. S. Hahm, P. H. Diamond, O. D. Gurcan, and G. Rewoldt, "Turbulent equipartition theory of toroidal momentum pincha)," Phys. Plasmas 15(5), 055902 (2008).

${ }^{20}$ X. Garbet, L. Garzotti, P. Mantica, H. Nordman, M. Valovic, H. Weisen, and C. Angioni, "Turbulent particle transport in magnetized plasmas," Phys. Rev. Lett. 91(3), 035001 (2003).

${ }^{21}$ W. Horton, "Drift waves and transport," Rev. Mod. Phys. 71(3), 735 (1999).

${ }^{22}$ G. E. Karniadakis, M. Israeli, and S. A. Orszag, "High-order splitting methods for the incompressible Navier-Stokes equations," J. Comput. Phys. 97(2), 414-443 (1991).

${ }^{23}$ J. E. Rice, C. Gao, M. L. Reinke, P. H. Diamond, N. T. Howard, H. J. Sun, I. Cziegler, A. E. Hubbard, Y. A. Podpaly, W. L. Rowan et al., "Non-local heat transport, rotation reversals and up/down impurity density asymmetries in Alcator C-Mod ohmic L-mode plasmas," Nucl. Fusion 53(3), 033004 (2013).

${ }^{24}$ P. Mantica, G. Gorini, F. Imbeaux, J. Kinsey, Y. Sarazin, R. Budny, I. Coffey, R. Dux, X. Garbet, L. Garzotti et al., "Perturbative transport experiments in JET low or reverse magnetic shear plasmas," Plasma Phys. Controlled Fusion 44(10), 2185 (2002).

${ }^{25}$ K. W. Gentle, R. V. Bravenec, G. Cima, H. Gasquet, G. A. Hallock, P. E. Phillips, D. W. Ross, W. L. Rowan, A. J. Wootton, T. P. Crowley et al., "An experimental counter-example to the local transport paradigm," Phys. Plasmas 2(6), 2292-2298 (1995).

${ }^{26}$ M. Greenwald, A. Bader, S. Baek, H. Barnard, W. Beck, W. Bergerson, I. Bespamyatnov, M. Bitter, P. Bonoli, M. Brookman et al., "Overview of experimental results and code validation activities at Alcator C-Mod," Nucl. Fusion 53(10), 104004 (2013).

${ }^{27}$ D. Del-Castillo-Negrete, P. Mantica, V. Naulin, J. J. Rasmussen, and JET EFDA contributors, "Non-local models of perturbative transport: numerical results and application to JET experiments," Nucl. Fusion 48, 075009 (2008).

${ }^{28} \mathrm{Z}$. Lin and T. S. Hahm, "Turbulence spreading and transport scaling in global gyrokinetic particle simulations," Phys. Plasmas 11(3), 1099-1108 (2004).

${ }^{29}$ T. S. Hahm, P. H. Diamond, O. D. Gurcan, and G. Rewoldt, "Nonlinear gyrokinetic theory of toroidal momentum pinch," Phys. Plasmas 14(7), 072302 (2007)

${ }^{30}$ S. Yi, J. M. Kwon, P. H. Diamond, and T. S. Hahm, "Turbulence spreading as a non-local mechanism of global confinement degradation and ion temperature profile stiffness," Nucl. Fusion 55(9), 092002 (2015). 